\title{
Euler sums with Dirichlet characters
}

\author{
by
}

Minking Eie and Wen-Chin Liaw (Minhsiung)

1. Introduction and statement of the main result. For a pair of positive integers $p$ and $q$ with $q>1$, the classical Euler sum is defined as

$$
S_{p, q}:=\sum_{k=1}^{\infty} \frac{1}{k^{q}} \sum_{j=1}^{k} \frac{1}{j^{p}} .
$$

The number $w=p+q$ is called the weight of $S_{p, q}$. The evaluations of $S_{p, q}$ in terms of values at positive integers of the Riemann zeta function are known when $p=1$ or $(p, q)=(2,4)$ or $(p, q)=(4,2)$ or $p=q$ or $p+q$ is odd. The following theorems are classical and both due to Euler $[10]$.

TheOREM A. For each positive integer $n$ with $n \geq 2$, we have

$$
S_{1, n}=\frac{n+2}{2} \zeta(n+1)-\frac{1}{2} \sum_{l=2}^{n-1} \zeta(l) \zeta(n+1-l) .
$$

Here $\zeta(s)$ is the Riemann zeta function defined by

$$
\zeta(s):=\sum_{n=1}^{\infty} \frac{1}{n^{s}}, \quad \operatorname{Re} s>1 .
$$

TheOREM B. For a pair of positive integers $p$ and $q$ with $p, q \geq 2$ and an odd weight $w=p+q$, we have

2000 Mathematics Subject Classification: Primary 11M06; Secondary 11B68, 11M35.

Key words and phrases: Euler sums, Dirichlet characters, Kronecker limit formula, Hurwitz zeta function.

The first author was supported by the Department of Mathematics, National Chung Cheng University and by the National Science Council of Taiwan, Republic of China.

The second author was partially supported by grant NSC93-2115-M-194-015 from the National Science Council of Taiwan, Republic of China. 


$$
\begin{aligned}
S_{p, q}= & \frac{1}{2} \zeta(w)+\frac{1-(-1)^{p}}{2} \zeta(p) \zeta(q) \\
& +(-1)^{p} \sum_{l=0}^{[p / 2]}\left(\begin{array}{c}
w-2 l-1 \\
q-1
\end{array}\right) \zeta(2 l) \zeta(w-2 l) \\
& +(-1)^{p} \sum_{l=0}^{[q / 2]}\left(\begin{array}{c}
w-2 l-1 \\
p-1
\end{array}\right) \zeta(2 l) \zeta(w-2 l) .
\end{aligned}
$$

Here $\zeta(s)$ is the Riemann zeta function and $\zeta(0)=-1 / 2$.

The evaluation of Euler sums has a long history. The problem of evaluating such sums was first proposed in 1742 in a letter from Goldbach to Euler. In 1775, Euler [10] himself proved Theorem A, but gave (an incorrect version of) Theorem B without proof. By using a partial fraction decomposition, Euler first derived linear relations among Euler sums of the same weight and then obtained his evaluation of $S_{1, n}$ by solving a system of linear equations. He then computed many examples for weight not exceeding 13 and conjectured the general formula. N. Nielsen [13] was the first to fill in the gap by giving the correct version and a proof. The sums $S_{p, q}$ have since been re-evaluated time and again $[2,6,11,16]$. For a discussion of earlier works and references prior to 1985 , the readers are referred to [1, pp. 252-253].

There are many interesting generalizations for the classical (double) Euler sums, for instance, extended or truncated double sums [7], alternating double sums [9], or sums on arithmetic progressions [8]. There are also generalizations to multiple zeta values $[4,12,19]$ or $k$-fold sums [3] in connection with Vassiliev knot invariants of knot theory and Feynman diagrams of perturbative quantum field theory in physics (see especially $[4,19]$ and references therein).

In this paper, we shall follow Euler's original approach, which was also taken up in $[2,4,12,13]$, to study another generalization of Euler sums, to which we refer as "Euler sums with Dirichlet characters." There is also recent work $[5,14,15]$ in this direction.

Recall that (see [17]) a Dirichlet character modulo $N$ is a multiplicative group homomorphism $\chi=\chi_{N}:(\mathbb{Z} / N \mathbb{Z})^{*} \rightarrow \mathbb{C}^{*}$ and extended to $\mathbb{Z}$ by letting $\chi(a)=0$ if $\operatorname{gcd}(a, N)>1$. Note that $\chi$ is then periodic of period $N$. Also, $\chi$ is called even if $\chi(-1)=1$ and odd if $\chi(-1)=-1$.

Now we are ready to define Euler sums with Dirichlet characters. For a pair of positive integers $p$ and $q$ with $q \geq 2$ and a Dirichlet character $\chi$ modulo $N$, we define

$$
S_{p, q}^{\chi}:=\sum_{k=1}^{\infty} \frac{\chi(k)}{k^{q}} \sum_{j=1}^{k} \frac{1}{j^{p}} .
$$

Here is our new theorem concerning the evaluation of $S_{p, q}^{\chi}$. 
MAIN TheORem. Given a pair of positive integers $p$ and $q$ with $q \geq 2$ and a Dirichlet character $\chi$ modulo $N$. Then the sum $S_{p, q}^{\chi}$ defined in (1.4) can be evaluated in terms of values at positive integers of Hurwitz zeta functions either when $\chi$ is even and the weight $p+q$ is odd, or when $\chi$ is odd and the weight $p+q$ is even.

Remark. Although we state the Main Theorem only for the sum $S_{p, q}^{\chi}$, it holds for a more general double Euler sum with two Dirichlet characters. See the concluding remark at the end of the paper.

This sheds some light on the reason why such evaluations exist in the case of classical Euler sums. It also unfolds the other side that Euler had the opportunity but missed to discover.

Indeed, the theory we develop here can be applied to determine whether an analogous Euler sum can be evaluated or not. For example, the extended or truncated Euler sums

$$
E_{p, q}^{(n)}:=\sum_{k=1}^{\infty} \frac{1}{k^{q}} \sum_{j=1}^{n k} \frac{1}{j^{p}}, \quad T_{p, q}^{(n)}:=\sum_{k=1}^{\infty} \frac{1}{k^{q}} \sum_{j=1}^{[k / n]} \frac{1}{j^{p}},
$$

as considered in [7], both can be evaluated when the weight $p+q$ is odd. On the other hand, the alternating Euler sum

$$
G_{p, q}^{+-}:=\sum_{k=0}^{\infty} \frac{(-1)^{k}}{(2 k+1)^{q}} \sum_{j=1}^{k} \frac{1}{j^{p}},
$$

as considered in [9], can be evaluated when $p+q$ is even.

The organization of the paper is as follows. In Section 2 we decompose $S_{p, q}^{\chi}$ into elementary building blocks $H_{p, q}(a / N, b / N)$, derive basic properties of these building blocks, and develop relations among $H_{1, n}(a / N, b / N)$. Section 3 contains algebraic preliminaries. The proof of the Main Theorem is divided into two cases according to the parity of the characters. We deal with Euler sums with even characters in Section 4. The case of odd characters is harder, and is treated in Section 5.

2. Elementary building blocks of Euler sums. Throughout the paper, we will fix a positive integer $N$ and a Dirichlet character $\chi$ of conductor $N$. For a pair of positive integers $p$ and $q$ with $q \geq 2$ and another pair of positive integers $a$ and $b$ with $a, b \leq N$, we define

$$
H_{p, q}\left(\frac{a}{N}, \frac{b}{N}\right):=\sum_{k=0}^{\infty} \frac{1}{(k N+b)^{q}} \sum_{j=0}^{\widetilde{k}} \frac{1}{(j N+a)^{p}},
$$


where

$$
\widetilde{k}= \begin{cases}k & \text { if } a \leq b, \\ k-1 & \text { if } a>b .\end{cases}
$$

Such kind of sums were first introduced in [8] in an attempt to produce analogues of classical Euler sums. They play a role of "building blocks" or "atoms" in expressing various kinds of Euler sums. For example, we have

$$
\begin{aligned}
S_{p, q} & =\sum_{a=1}^{N} \sum_{b=1}^{N} H_{p, q}\left(\frac{a}{N}, \frac{b}{N}\right), \\
S_{p, q}^{\chi} & =\sum_{a=1}^{N} \sum_{b=1}^{N} \chi(b) H_{p, q}\left(\frac{a}{N}, \frac{b}{N}\right), \\
E_{p, q}^{(N)} & =\sum_{a=1}^{N} N^{q} H_{p, q}\left(\frac{a}{N}, \frac{N}{N}\right), \\
T_{p, q}^{(N)} & =\sum_{b=1}^{N} N^{p} H_{p, q}\left(\frac{N}{N}, \frac{b}{N}\right) .
\end{aligned}
$$

The sums $H_{p, q}(a / N, b / N)$ satisfy the following reflection formulce when $p, q \geq 2$ :

$$
H_{p, q}\left(\frac{a}{N}, \frac{b}{N}\right)+H_{q, p}\left(\frac{b}{N}, \frac{a}{N}\right)=N^{-(p+q)} \zeta\left(p, \frac{a}{N}\right) \zeta\left(q, \frac{b}{N}\right)
$$

when $a \neq b$, and

$$
\begin{aligned}
H_{p, q}\left(\frac{a}{N}, \frac{a}{N}\right) & +H_{q, p}\left(\frac{a}{N}, \frac{a}{N}\right) \\
& =N^{-(p+q)}\left\{\zeta\left(p, \frac{a}{N}\right) \zeta\left(q, \frac{a}{N}\right)+\zeta\left(p+q, \frac{a}{N}\right)\right\} .
\end{aligned}
$$

Not all of $H_{p, q}(a / N, b / N)$ can be evaluated individually even when the weight $p+q$ is odd. However, it turns out that linear combinations such as

$$
H_{p, q}(a / N, b / N) \pm H_{p, q}((N-a) / N,(N-b) / N)
$$

can be evaluated, and so can the sum $S_{p, q}^{\chi}$, as we shall see in Sections 4 and 5 . Here we begin with the cases when $p=1$.

The Kronecker limit formula for the Hurwitz zeta function is given by [18, p. $271, \S 13.21]$

$$
\lim _{s \rightarrow 1^{+}}\left\{\zeta(s, x)-\frac{1}{s-1}\right\}=-\frac{\Gamma^{\prime}(x)}{\Gamma(x)},
$$


where $\zeta(s, x)$ is the Hurwitz zeta function and $\Gamma(x)$ is the well-known gamma function. For convenience' sake, we shall employ the familiar notation

$$
\psi(x):=\frac{\Gamma^{\prime}(x)}{\Gamma(x)} .
$$

The Kronecker limit formula has the following corollary:

$$
\sum_{j=0}^{\infty}\left\{\frac{1}{j N+a}-\frac{1}{j N+b}\right\}=\frac{1}{N}\left\{-\psi\left(\frac{a}{N}\right)+\psi\left(\frac{b}{N}\right)\right\},
$$

valid for positive integers $a$ and $b$. For $x>0$, we also have

$$
\psi(x+1)=\frac{1}{x}+\psi(x),
$$

a direct consequence of the functional equation of the gamma function.

Now we are ready to develop relations among $H_{1, n}(a / N, b / N)$.

Proposition 2.1. For a positive integer a with $a<N$ and a positive integer $n$ with $n \geq 2$, we have

$$
\begin{aligned}
N^{n+1} H_{1, n}\left(\frac{N}{N}, \frac{a}{N}\right)= & \frac{n}{2} \zeta\left(n+1, \frac{a}{N}\right)+\zeta\left(n, \frac{a}{N}\right)\left\{\psi\left(\frac{a}{N}\right)+\gamma\right\} \\
& -\frac{1}{2} \sum_{l=2}^{n-1} \zeta\left(l, \frac{a}{N}\right) \zeta\left(n+1-l, \frac{a}{N}\right)
\end{aligned}
$$

Proof. By definition, we have

$$
N^{n+1} H_{1, n}\left(\frac{N}{N}, \frac{a}{N}\right)=N^{n+1} \sum_{k=0}^{\infty} \frac{1}{(k N+a)^{n}} \sum_{j=1}^{k} \frac{1}{j N}=\sum_{k=0}^{\infty} \frac{1}{(k+r)^{n}} \sum_{j=1}^{k} \frac{1}{j},
$$

where $r=a / N$. Rewrite the above series as

$$
\sum_{k=0}^{\infty} \sum_{j=1}^{\infty} \frac{1}{(k+j+r)^{n} j}
$$

with a change of variable $k=k^{\prime}+j$ and still using $k$ instead of $k^{\prime}$. In the light of the partial fraction decomposition

$$
\frac{1}{(x+\alpha)^{n} x}=\frac{1}{\alpha^{n}}\left\{\frac{1}{x}-\frac{1}{x+\alpha}\right\}-\sum_{l=1}^{n-1} \frac{1}{\alpha^{l}(x+\alpha)^{n+1-l}},
$$

we are able to rewrite $N^{n+1} H_{1, n}(N / N, a / N)$ as

$$
\sum_{k=0}^{\infty} \frac{1}{(k+r)^{n}} \sum_{j=1}^{\infty}\left\{\frac{1}{j}-\frac{1}{k+j+r}\right\}-\sum_{l=1}^{\infty} \sum_{k=0}^{\infty} \sum_{j=1}^{\infty} \frac{1}{(k+r)^{l}(k+j+r)^{n+1-l}} .
$$


The first term in the above is equal to

or as

$$
\sum_{k=0}^{\infty} \frac{1}{(k+r)^{n}}\{\psi(k+1+r)+\gamma\},
$$

$$
N^{n+1} H_{1, n}\left(\frac{a}{N}, \frac{a}{N}\right)+\zeta\left(n, \frac{a}{N}\right)\left\{\psi\left(\frac{a}{N}\right)+\gamma\right\}
$$

by repeatedly using (2.12). The second term is equal to

$$
\begin{aligned}
-\sum_{l=1}^{n-1}\left\{\sum_{k=0}^{\infty} \sum_{j=0}^{\infty} \frac{1}{(k+r)^{l}(k+j+r)^{n+1-l}}-\zeta(n+1, r)\right\} \\
=-N^{n+1} H_{1, n}\left(\frac{a}{N}, \frac{a}{N}\right) \\
-N^{n+1} \sum_{l=2}^{n-1} H_{l, n+1-l}\left(\frac{a}{N}, \frac{a}{N}\right)+(n-1) \zeta\left(n+1, \frac{a}{N}\right) .
\end{aligned}
$$

With the reflection formula (2.7), the above is equal to

$$
\begin{aligned}
\frac{n}{2} \zeta\left(n+1, \frac{a}{N}\right)-N^{n+1} H_{1, n} & \left(\frac{a}{N}, \frac{a}{N}\right) \\
& -\frac{1}{2} \sum_{l=2}^{n-2} \zeta\left(l, \frac{a}{N}\right) \zeta\left(n+1-l, \frac{a}{N}\right) .
\end{aligned}
$$

Our assertion then follows from the addition of (2.15) and (2.16).

In a similar way, we obtain the following propositions.

Proposition 2.2. For positive integers $a$ and $b$ with $1 \leq a<b<N$ and a positive integer $n \geq 2$, we have

$$
\begin{aligned}
N^{n+1}\left\{H_{1, n}\left(\frac{a}{N}, \frac{b}{N}\right)\right. & \left.+H_{1, n}\left(\frac{N-a}{N}, \frac{b-a}{N}\right)\right\} \\
= & \zeta\left(n, \frac{b-a}{N}\right)\left\{-\psi\left(\frac{a}{N}\right)+\psi\left(\frac{b}{N}\right)\right\} \\
& +\zeta\left(n, \frac{b}{N}\right)\left\{-\psi\left(1-\frac{a}{N}\right)+\psi\left(\frac{b-a}{N}\right)\right\} \\
& -\sum_{l=2}^{n-1} \zeta\left(l, \frac{b}{N}\right) \zeta\left(n+1-l, \frac{b-a}{N}\right) .
\end{aligned}
$$


Proposition 2.3. For a positive integer a with $a<N$ and a positive integer $n \geq 2$, we have

$$
\begin{aligned}
& \text { (2.18) } \quad N^{n+1}\left\{H_{1, n}\left(\frac{a}{N}, \frac{a}{N}\right)+H_{1, n}\left(\frac{N-a}{N}, \frac{N}{N}\right)\right\} \\
& =\zeta\left(n+1, \frac{a}{N}\right)-\zeta\left(n, \frac{a}{N}\right)\left\{\psi\left(1-\frac{a}{N}\right)+\gamma\right\}-\sum_{l=2}^{n-1} \zeta(l) \zeta\left(n+1-l, \frac{a}{N}\right) .
\end{aligned}
$$

3. Algebraic preliminaries. In the sections that follow, we shall need the following identities on binomial coefficients, whose proofs are easy and may be found, e.g., in $[2,4]$.

Lemma 3.1. For $0 \leq \mu, \nu \leq m$,

$$
\sum_{k=0}^{m}(-1)^{k}\left(\begin{array}{c}
m-k \\
\nu
\end{array}\right)\left(\begin{array}{l}
\mu \\
k
\end{array}\right)=\left(\begin{array}{l}
m-\mu \\
m-\nu
\end{array}\right) .
$$

In particular, setting $\mu=m$ gives

$$
\sum_{k=0}^{m}(-1)^{k}\left(\begin{array}{c}
m-k \\
\nu
\end{array}\right)\left(\begin{array}{c}
m \\
k
\end{array}\right)=(-1)^{m} \delta_{m \nu} .
$$

We shall consider matrices of dimension $r$, i.e., $r$ by $r$ matrices, where $r=w-3$, and $w=p+q$ is the weight of Euler sums.

Notation 3.2. Let $I$ be the identity matrix of dimension $r, B=$ $\left[B_{i, j}\right]_{1 \leq i, j \leq r}$ with

$$
B_{i, j}:=(-1)^{i+1}\left(\begin{array}{l}
j \\
i
\end{array}\right)
$$

and $J$ be the permutation matrix of dimension $r$ with 1 on the reverse diagonal and 0 elsewhere, viz.,

$$
J=\left[\begin{array}{ccccc}
0 & 0 & \cdots & 0 & 1 \\
0 & 0 & \cdots & 1 & 0 \\
\vdots & \vdots & \ddots & \vdots & \vdots \\
1 & 0 & \cdots & 0 & 0
\end{array}\right] .
$$

LEMma 3.3. We have

(i) $J^{2}=B^{2}=I$ for any $r$.

(ii) $(J B)^{2}+(B J)^{2}=J B+B J$ if $r$ is even (i.e., if $w$ is odd).

(iii) $(J B)^{2}-(B J)^{2}=J B-B J$ if $r$ is odd (i.e., if $w$ is even). 
Proof. Denote by $(A)_{i j}$ the $(i, j)$ entry of a matrix $A$. Note that $(J)_{i j}=$ $\delta_{i+j, r+1}$, where $\delta$ is the Kronecker delta. An easy calculation using (3.2) shows that $J^{2}=B^{2}=I$. Note that multiplying $J$ from the left (resp. right) has the effect of changing the index $i \mapsto r+1-i$ (resp. $j \mapsto r+1-j$ ). It follows that

$$
(J B)_{i j}=(-1)^{r-i}\left(\begin{array}{c}
j \\
r+1-i
\end{array}\right), \quad(B J)_{i j}=(-1)^{i-1}\left(\begin{array}{c}
r+1-j \\
i
\end{array}\right),
$$

and hence

$$
\left(J B+(-1)^{r} B J\right)_{i j}=(-1)^{r-i}\left\{\left(\begin{array}{c}
j \\
r+1-i
\end{array}\right)-\left(\begin{array}{c}
r+1-j \\
i
\end{array}\right)\right\} .
$$

On the other hand, by (3.1),

$$
\begin{aligned}
\left((J B)^{2}\right)_{i j} & =\sum_{k=1}^{r}(-1)^{r-i}\left(\begin{array}{c}
k \\
r+1-i
\end{array}\right)(-1)^{r-k}\left(\begin{array}{c}
j \\
r+1-k
\end{array}\right) \\
& =(-1)^{r+1-i} \sum_{k=1}^{r}(-1)^{k}\left(\begin{array}{c}
r+1-k \\
r+1-i
\end{array}\right)\left(\begin{array}{l}
j \\
k
\end{array}\right) \\
& =(-1)^{r+1-i}\left\{\left(\begin{array}{c}
r+1-j \\
i
\end{array}\right)-\left(\begin{array}{c}
r+1 \\
i
\end{array}\right)\right\} .
\end{aligned}
$$

Hence,

$$
\left((B J)^{2}\right)_{i j}=\left(J(J B)^{2} J\right)_{i j}=(-1)^{i}\left\{\left(\begin{array}{c}
j \\
r+1-i
\end{array}\right)-\left(\begin{array}{c}
r+1 \\
r+1-i
\end{array}\right)\right\},
$$

and

$$
\left((J B)^{2}+(-1)^{r}(B J)^{2}\right)_{i j}=(-1)^{r-i}\left\{\left(\begin{array}{c}
j \\
r+1-i
\end{array}\right)-\left(\begin{array}{c}
r+1-j \\
i
\end{array}\right)\right\} .
$$

Parts (ii) and (iii) then follow from (3.3) and (3.4).

Corollary 3.4. Let $U=J B+B J$ and $V=J+B$.

(i) If $r$ is even, then $(U+I)(U-2 I)=0$ and $\left(V^{2}-I\right)\left(V^{2}-4 I\right)=0$. In particular, $U$ and $V$ are invertible in this case.

(ii) If $r$ is odd, then $(U-I)\left(U^{2}-4 I\right)=0$ and $V^{2}\left(V^{2}-3 I\right)\left(V^{2}-4 I\right)=0$.

Proof. Suppose the dimension $r$ is even. Then by Lemma 3.3(i),(ii), we have $U^{2}=(J B)^{2}+(B J)^{2}+2 I=J B+B J+2 I=U+2 I$. Since $U$ satisfies a polynomial equation with a nonzero constant term, $U$ is invertible. Note that $V^{2}=J B+B J+2 I=U+2 I$. We have $\left(V^{2}-I\right)\left(V^{2}-4 I\right)=0$, whence $V$ is invertible. Now suppose $r$ is odd. By Lemma 3.3(i),(iii),

$$
\begin{aligned}
(J B)^{3}+(B J)^{3} & =(J B-B J)\left((J B)^{2}-(B J)^{2}\right)+(J B+B J) \\
& =(J B-B J)^{2}+(J B+B J)=(J B+B J)^{2}+(J B+B J)-4 I \\
& =U^{2}+U-4 I .
\end{aligned}
$$


Consequently,

$$
U^{3}=(J B)^{3}+(B J)^{3}+3(J B+B J)=U^{2}+4 U-4 I,
$$

or $(U-I)\left(U^{2}-4 I\right)=0$. Since $V^{2}=U+2$, the last assertion follows.

REMARK. With a more careful analysis, one can show that if $r$ is odd then $V(V-2 I)\left(V^{2}-3 I\right)=0$, but we will not need this.

4. Euler sums with even characters. For Euler sums with even Dirichlet characters, we shall consider those of odd weight. These include the classical Euler sums $S_{p, q}$ evaluated in Theorems A and B. As mentioned in Section 1, the evaluations of $S_{p, q}$ with odd weight $p+q$ were initially obtained by solving a system of linear equations. Linear relations among Euler sums of the same weight can be easily derived from the partial fraction decomposition (see [2, 4, 12] or even [10]):

$$
\begin{aligned}
& \frac{1}{(x+\alpha)^{q} x^{p}} \\
& =\sum_{l=2}^{p} \frac{(-1)^{p} A_{l}}{\alpha^{w-l} x^{l}}+\sum_{l=2}^{q} \frac{(-1)^{p} B_{l}}{\alpha^{w-l}(x+\alpha)^{l}}+\frac{(-1)^{p} A_{1}}{\alpha^{w-1}}\left\{\frac{1}{x}-\frac{1}{x+\alpha}\right\},
\end{aligned}
$$

where $w=p+q$,

$$
A_{l}:=A_{l}^{p, q}=(-1)^{l}\left(\begin{array}{c}
w-l-1 \\
q-1
\end{array}\right) \quad \text { and } \quad B_{l}:=B_{l}^{p, q}=\left(\begin{array}{c}
w-l-1 \\
p-1
\end{array}\right) .
$$

Note that $A_{1}=-B_{1}$. Setting $x=j$ and $\alpha=k$ and summing over all positive integers $j$ and $k$, we get

$$
\begin{aligned}
S_{p, q}-\zeta(p+q)= & \sum_{l=2}^{p}(-1)^{p} A_{l} \zeta(w-l) \zeta(l) \\
& +\sum_{l=2}^{q}(-1)^{p} B_{l}\left\{S_{w-l, l}-\zeta(p+q)\right\} \\
& +(-1)^{p} A_{1} S_{1, w-1} .
\end{aligned}
$$

Now fix $p+q=2 n+1$ with $n \geq 2$; then we have the following linear relations among $S_{2,2 n-1}, S_{3,2 n-2}, \ldots, S_{2 n-1,2}$ :

$$
\left\{\begin{array}{l}
S_{2,2 n-1}=S_{2,2 n-1}+2 S_{3,2 n-2}+3 S_{4,2 n-3}+\cdots+(2 n-2) S_{2 n-1,2}+\Delta(2), \\
S_{3,2 n-2}=-S_{3,2 n-2}-3 S_{4,2 n-3}-\cdots-\left(\begin{array}{c}
2 n-2 \\
2
\end{array}\right) S_{2 n-1,2}+\Delta(3), \\
S_{4,2 n-3}=S_{4,2 n-3}+\cdots+\left(\begin{array}{c}
2 n-2 \\
3
\end{array}\right) S_{2 n-1,2}+\Delta(4) \\
\cdots \\
S_{2 n-2,3}=S_{2 n-2,3}+(2 n-2) S_{2 n-1,2}+\Delta(2 n-2) \\
S_{2 n-1,2}=-S_{2 n-1,2}+\Delta(2 n-1)
\end{array}\right.
$$


where $\Delta(j), j=2, \ldots, 2 n-1$, are known values in $S_{1,2 n}$ and values of the Riemann zeta function. The matrix of coefficients of the above system of equations has size $(2 n-2) \times(2 n-2)$ and rank $n-1$. Of course, this is not enough to determine the values of $S_{2,2 n-1}, S_{3,2 n-2}, \ldots, S_{2 n-1,2}$ uniquely. Fortunately, we still have reflection formulæ given by

$$
S_{p, q}+S_{q, p}=\zeta(p) \zeta(q)+\zeta(p+q)
$$

for $p, q \geq 2$. This adds $n-1$ more linear equations to the system (4.4) so that $S_{p, q}$ can be determined uniquely. Indeed, let $X={ }^{t}\left(S_{2,2 n-1}, S_{3,2 n-2}\right.$, $\left.\ldots, S_{2 n-1,2}\right)$ be a column vector consisting of unknowns. Then (4.4) and (4.5) can be put in matrix form as

$$
\begin{gathered}
X-B X=\Delta, \\
X+J X=\mathbf{z},
\end{gathered}
$$

where $J$ and $B$ are matrices of dimension $2 n-2$ as defined in Notation 3.2, and where $\Delta={ }^{t}(\Delta(j))_{j=2, \ldots, 2 n-1}$ and $\mathbf{z}={ }^{t}(\zeta(p) \zeta(2 n+1-p)+$ $\zeta(2 n+1))_{p=2, \ldots, 2 n-1}$ are column vectors with known entries. Subtraction of (4.6) from (4.7) gives a system of linear equations $(J+B) X=\mathbf{z}-\Delta$, and by Corollary 3.4(i), the coefficient matrix $J+B$ is invertible since the weight $p+q=2 n+1$ is odd.

Different substitutions of $x$ and $j$ into (4.1) lead to different linear relations among $H_{p, q}(a / N, b / N)$ for various $p$ and $q$.

Proposition 4.1. For a positive integer a with $2 a<N$, let

$$
K_{p, q}(a, N):=H_{p, q}\left(\frac{a}{N}, \frac{2 a}{N}\right)+H_{p, q}\left(\frac{N-a}{N}, \frac{N-2 a}{N}\right) .
$$

Then for positive integers $p$ and $q$ with $p, q \geq 2$ and $w=p+q$, we have

$$
K_{p, q}(a, N)=(-1)^{p} \sum_{l=2}^{q} B_{l} K_{w-l, l}(a, N)+k_{p, q}(a, N),
$$

where

$$
\begin{aligned}
& k_{p, q}(a, N) \\
& =\sum_{l=2}^{p}(-1)^{p} A_{l} N^{-w}\left\{\zeta\left(l, \frac{a}{N}\right) \zeta\left(w-l, \frac{a}{N}\right)+\zeta\left(l, \frac{N-a}{N}\right) \zeta\left(w-l, \frac{N-a}{N}\right)\right\} \\
& +(-1)^{p} A_{1} N^{-w}\left\{\zeta\left(w-1, \frac{a}{N}\right)-\zeta\left(w-1, \frac{N-a}{N}\right)\right\}\left\{\pi \cot \frac{\pi a}{N}-\pi \cot \frac{2 \pi a}{N}\right\} \\
& +(-1)^{p+1} A_{1} N^{-w} \sum_{l=2}^{w-2} \zeta\left(l, \frac{N-a}{N}\right) \zeta\left(w-l, \frac{a}{N}\right) .
\end{aligned}
$$


Proof. Set $x=a j+b$ and $\alpha=a k+b$ and sum over all non-negative integers $j$ and $k$ to get

$$
\begin{aligned}
H_{p, q}\left(\frac{a}{N}, \frac{2 a}{N}\right) & =(-1)^{p} \sum_{l=2}^{q} B_{l} H_{w-l, l}\left(\frac{a}{N}, \frac{2 a}{N}\right) \\
+ & (-1)^{p} \sum_{l=2}^{q} A_{l} N^{-w} \zeta\left(w-l, \frac{a}{N}\right) \zeta\left(l, \frac{a}{N}\right) \\
+ & (-1)^{p} A_{1} N^{-w} \zeta\left(w-1, \frac{a}{N}\right)\left\{-\psi\left(\frac{a}{N}\right)+\psi\left(\frac{2 a}{N}\right)\right\} \\
+ & (-1)^{p} A_{1} H_{1, w-1}\left(\frac{2 a}{N}, \frac{a}{N}\right) .
\end{aligned}
$$

Replacing $a$ by $N-a$, we get

$$
\begin{aligned}
& H_{p, q}\left(\frac{N-a}{N}, \frac{N-2 a}{N}\right)=(-1)^{p} \sum_{l=2}^{q} B_{l} H_{w-l, l}\left(\frac{N-a}{N}, \frac{N-2 a}{N}\right) \\
& +(-1)^{p} \sum_{l=2}^{q} A_{l} N^{-w} \zeta\left(w-l, \frac{N-a}{N}\right) \zeta\left(l, \frac{N-a}{N}\right) \\
& +(-1)^{p} A_{1} N^{-w} \zeta\left(w-1, \frac{N-a}{N}\right)\left\{-\psi\left(\frac{N-a}{N}\right)+\psi\left(\frac{N-2 a}{N}\right)\right\} \\
& +(-1)^{p} A_{1} H_{1, w-1}\left(\frac{N-2 a}{N}, \frac{N-a}{N}\right) .
\end{aligned}
$$

Our assertion then follows from the combination of (4.10) and (4.11), and the evaluation

$$
H_{1, n}\left(\frac{2 a}{N}, \frac{a}{N}\right)+H_{1, w-1}\left(\frac{N-2 a}{N}, \frac{N-a}{N}\right)
$$

as given in Proposition 2.2.

Note the resemblance between (4.9) and (4.3). Just as in the case of $S_{p, q}$, utilizing relations in (4.9) with $p=2, \ldots, w-2$ is not sufficient to uniquely determine $K_{p, q}(a, N)$. Another obstacle is the lack of reflection formulæ (4.5). It is therefore necessary to introduce two more companion families. Define

$$
\begin{aligned}
L_{p, q}(a, N) & :=H_{p, q}\left(\frac{2 a}{N}, \frac{a}{N}\right)+H_{p, q}\left(\frac{N-2 a}{N}, \frac{N-a}{N}\right), \\
M_{p, q}(a, N) & :=M_{p, q}\left(\frac{a}{N}, \frac{N-a}{N}\right)+H_{p, q}\left(\frac{N-a}{N}, \frac{a}{N}\right) .
\end{aligned}
$$


In what follows, we use the abbreviations $K_{p, q}, L_{p, q}$ and $M_{p, q}$ for $K_{p, q}(a, N), L_{p, q}(a, N)$ and $M_{p, q}(a, N)$, respectively.

From the above definitions, we see immediately the following reflection formulæ for $p, q \geq 2$ :

$$
\begin{aligned}
K_{p, q}+ & L_{q, p} \\
& =N^{-w}\left\{\zeta\left(p, \frac{a}{N}\right) \zeta\left(q, \frac{2 a}{N}\right)+\zeta\left(p, \frac{N-a}{N}\right) \zeta\left(q, \frac{N-a}{N}\right)\right\}, \\
M_{p, q}+ & M_{q, p} \\
& =N^{-w}\left\{\zeta\left(p, \frac{a}{N}\right) \zeta\left(q, \frac{N-a}{N}\right)+\zeta\left(p, \frac{N-a}{N}\right) \zeta\left(q, \frac{a}{N}\right)\right\} .
\end{aligned}
$$

Also we have the following relations by a similar consideration:

$$
\begin{aligned}
L_{p, q} & =(-1)^{p} \sum_{l=2}^{q} B_{l} M_{w-l, l}+(-1)^{p} A_{1} M_{1, w-1}+\ell_{p, q}, \\
M_{p, q} & =(-1)^{p} \sum_{l=2}^{q} B_{l} L_{w-l, l}+(-1)^{p} A_{1} K_{1, w-1}+m_{p, q},
\end{aligned}
$$

where $\ell_{p, q}$ and $m_{p, q}$ are known values in Hurwitz zeta functions.

As a first step, we obtain the values of $K_{1, w-1}$ and $M_{1, w-1}$ through the relations when the weight $w$ is odd.

Proposition 4.2. For each positive integer $n, K_{1,2 n}$ and $M_{1,2 n}$ can be evaluated in terms of values at positive integers of Hurwitz zeta functions.

Proof. Note that

$$
\begin{aligned}
K_{1,2 n}+M_{1,2 n}= & \left\{H_{1,2 n}\left(\frac{a}{N}, \frac{2 a}{N}\right)+H_{1,2 n}\left(\frac{N-a}{N}, \frac{a}{N}\right)\right\} \\
& +\left\{H_{1,2 n}\left(\frac{a}{N}, \frac{N-a}{N}\right)+H_{1,2 n}\left(\frac{N-a}{N}, \frac{N-2 a}{N}\right)\right\} .
\end{aligned}
$$

According to Proposition 2.2, the value is equal to

$$
\begin{aligned}
& N^{-w} \zeta\left(2 n, \frac{a}{N}\right)\left\{-\psi\left(\frac{a}{N}\right)+\psi\left(\frac{2 a}{N}\right)\right\} \\
& +N^{-w} \zeta\left(2 n, \frac{N-a}{N}\right)\left\{-\psi\left(\frac{N-a}{N}\right)+\psi\left(\frac{N-2 a}{N}\right)\right\} \\
& +N^{-w}\left\{\zeta\left(2 n, \frac{N-2 a}{N}\right)-\zeta\left(2 n, \frac{2 a}{N}\right)\right\} \pi \cot \frac{a \pi}{N} \\
& -\sum_{l=2}^{2 n-1} N^{-w}\left\{\zeta\left(l, \frac{2 a}{N}\right) \zeta\left(2 n+1-l, \frac{a}{N}\right)+\zeta\left(l, \frac{N-a}{N}\right) \zeta\left(2 n+1-l, \frac{N-2 a}{N}\right)\right\} .
\end{aligned}
$$


On the other hand, set $q=2$ and $p=2 n-1$ in (4.16) and (4.17) to obtain

$$
\begin{aligned}
L_{2 n-1,2} & =-M_{2 n-1,2}+(2 n-1) M_{1,2 n}+\ell_{2 n-1,2}, \\
M_{2 n-1,2} & =-L_{2 n-1,2}+(2 n-1) K_{1,2 n}+m_{2 n-1,2} .
\end{aligned}
$$

It follows that

$$
(2 n-1)\left\{K_{1,2 n}-M_{1,2 n}\right\}=\ell_{2 n-1,2}-m_{2 n-1,2} .
$$

Our assertions then follow from (4.18) and (4.19).

Let $K, L, M$ denote, respectively, the column vectors consisting of three sets of variables

$$
\begin{gathered}
K_{2, w-2}, K_{3, w-3}, \ldots, K_{w-2,2} ; \quad L_{2, w-2}, L_{3, w-3}, \ldots, L_{w-2,2} \\
M_{2, w-2}, M_{3, w-3}, \ldots, M_{w-2,2}
\end{gathered}
$$

Suppose that

$$
w=p+q=2 n+1 \quad \text { and } \quad r=w-3=2 n-2
$$

with $n \geq 2$. Let $I, J, B$ be the $r \times r$ matrices defined in Notation 3.2. In matrix form, relations (4.9), (4.14), (4.15), (4.16) and (4.17) can be rewritten as

$$
\begin{aligned}
& K=B K+\mathbf{k}, \\
& L=B M+\mathbf{l}, \\
& M=B L+\mathbf{m}, \\
& K+J L=\mathbf{c}, \\
& M+J M=\mathbf{d},
\end{aligned}
$$

where $\mathbf{k}, \mathbf{l}, \mathbf{m}, \mathbf{c}, \mathbf{d}$ are column $(2 n-2) \times 1$ vectors consisting of known values.

From (2) and (4), we have

$$
K=\mathbf{c}-J L=-J B M+(\mathbf{c}-J \mathbf{l}) .
$$

Substituting (6) into (1), we get

$$
(B-I) J B M=(B-I)(\mathbf{c}-J \mathbf{l})+\mathbf{k} .
$$

The rank of $(B-I) J B$ is only $n-1$, but (5) provides another $n-1$ conditions for the unknown $M$. Consequently, we can solve for $M$ from (7) with the extra conditions (5), and the values of $L$ and $K$ then follow.

Indeed, if we consider instead $X=B M$ as a new variable vector, then (5) and (7) are equivalent to the system

$$
\left\{\begin{array}{l}
(B+J B) X=\mathbf{d}, \\
(J-B J) X=(I-B)(\mathbf{c}-J \mathbf{l})-\mathbf{k} .
\end{array}\right.
$$


So

$$
(J+B+J B-B J) X=(I-B)(\mathbf{c}-J \mathbf{l})+\mathbf{d}-\mathbf{k} .
$$

Let $P=(J+B)+(J B-B J)$ be the coefficient matrix of the above system. Then

$$
\begin{aligned}
P^{2} & =(J+B)^{2}+(J B-B J)^{2} \\
& =J B+B J+(J B)^{2}+(B J)^{2}=2(J B+B J) .
\end{aligned}
$$

By Corollary 3.4(i), $\operatorname{det} P^{2}=2^{r} \operatorname{det}(J B+B J) \neq 0$, proving that $P$ is invertible. Thus the solvability of the system is beyond doubt and this proves the following theorem.

TheOREM 4.3. For positive integer a with $2 a<N$, let

$$
\begin{aligned}
K_{p, q}(a, N) & :=H_{p, q}\left(\frac{a}{N}, \frac{2 a}{N}\right)+H_{p, q}\left(\frac{N-a}{N}, \frac{N-2 a}{N}\right), \\
L_{p, q}(a, N) & :=H_{p, q}\left(\frac{2 a}{N}, \frac{a}{N}\right)+H_{p, q}\left(\frac{N-2 a}{N}, \frac{a}{N}\right), \\
M_{p, q}(a, N) & :=H_{p, q}\left(\frac{a}{N}, \frac{N-a}{N}\right)+H_{p, q}\left(\frac{N-a}{N}, \frac{a}{N}\right) .
\end{aligned}
$$

Then $K_{p, q}(a, N), L_{p, q}(a, N)$ and $M_{p, q}(a, N)$ can be evaluated in terms of values at positive integers of Hurwitz zeta functions when the weight $p+q$ is odd.

We shall refer to the triple families $K, L$, and $M$ as the $K L M$-system for brevity. Some other triple families, the ETD-system and FGR-system, share the same kind of relations as (1)-(5), so we have the following immediate consequences.

Corollary 4.4. For positive integer a with $a<N$, let

$$
\begin{aligned}
E_{p, q}(a, N) & :=H_{p, q}\left(\frac{a}{N}, \frac{N}{N}\right)+H_{p, q}\left(\frac{N-a}{N}, \frac{N}{N}\right), \\
T_{p, q}(a, N) & :=H_{p, q}\left(\frac{N}{N}, \frac{a}{N}\right)+H_{p, q}\left(\frac{N}{N}, \frac{N-a}{N}\right), \\
D_{p, q}(a, N) & :=H_{p, q}\left(\frac{a}{N}, \frac{a}{N}\right)+H_{p, q}\left(\frac{N-a}{N}, \frac{N-a}{N}\right) .
\end{aligned}
$$

Then $E_{p, q}(a, N), T_{p, q}(a, N)$ and $D_{p, q}(a, N)$ can be evaluated in terms of values at positive integers of Hurwitz zeta functions when the weight $p+q$ is odd. 
Corollary 4.5. For positive integers $j, k$ and $a$ with $k a<N$ and $k \geq j+2, k \neq 2 j$, let

$$
\begin{aligned}
F_{p, q}(j, k ; a, N) & :=H_{p, q}\left(\frac{j a}{N}, \frac{k a}{N}\right)+H_{p, q}\left(\frac{(k-j) a}{N}, \frac{k a}{N}\right) \\
+ & H_{p, q}\left(\frac{N-j a}{N}, \frac{N-k a}{N}\right)+H_{p, q}\left(\frac{N-(k-j) a}{N}, \frac{N-k a}{N}\right), \\
G_{p, q}(j, k ; a, N):= & H_{p, q}\left(\frac{k a}{N}, \frac{j a}{N}\right)+H_{p, q}\left(\frac{k a}{N}, \frac{(k-j) a}{N}\right) \\
+ & H_{p, q}\left(\frac{N-k a}{N}, \frac{N-j a}{N}\right)+H_{p, q}\left(\frac{N-k a}{N}, \frac{N-(k-j) a}{N}\right), \\
R_{p, q}(j, k ; a, N):= & H_{p, q}\left(\frac{(k-j) a}{N}, \frac{N-j a}{N}\right)+H_{p, q}\left(\frac{N-j a}{N}, \frac{(k-j) a}{N}\right) \\
& \quad+H_{p, q}\left(\frac{j a}{N}, \frac{N-(k-j) a}{N}\right)+H_{p, q}\left(\frac{N-(k-j) a}{N}, \frac{j a}{N}\right) .
\end{aligned}
$$

Then $F_{p, q}(j, k ; a, N), G_{p, q}(j, k ; a, N)$ and $R_{p, q}(j, k ; a, N)$ can be evaluated in terms of values at positive integers of Hurwitz zeta functions when the weight $p+q$ is odd.

Recall that our goal is to evaluate the sum in (2.8). To do so, we shall show that the triple families $F_{p, q}(j, k ; a, N), G_{p, q}(j, k ; a, N)$ and $R_{p, q}(j, k ; a, N)$ with four members each can be further separated into subfamilies with just two members. Without loss of generality, we let $j=1$ and $k=3$ and consider new families given by

$$
\begin{aligned}
\widetilde{F}_{p, q}:= & H_{p, q}\left(\frac{a}{N}, \frac{3 a}{N}\right)+H_{p, q}\left(\frac{N-a}{N}, \frac{N-3 a}{N}\right) \\
& -H_{p, q}\left(\frac{2 a}{N}, \frac{3 a}{N}\right)-H_{p, q}\left(\frac{N-2 a}{N}, \frac{N-3 a}{N}\right), \\
\widetilde{G}_{p, q}:= & H_{p, q}\left(\frac{3 a}{N}, \frac{a}{N}\right)+H_{p, q}\left(\frac{N-3 a}{N}, \frac{N-a}{N}\right) \\
& -H_{p, q}\left(\frac{3 a}{N}, \frac{2 a}{N}\right)-H_{p, q}\left(\frac{N-3 a}{N}, \frac{N-2 a}{N}\right), \\
\widetilde{R}_{p, q}:= & H_{p, q}\left(\frac{a}{N}, \frac{N-2 a}{N}\right)+H_{p, q}\left(\frac{N-a}{N}, \frac{2 a}{N}\right) \\
& -H_{p, q}\left(\frac{2 a}{N}, \frac{N-a}{N}\right)-H_{p, q}\left(\frac{N-2 a}{N}, \frac{a}{N}\right) .
\end{aligned}
$$


Note that $\widetilde{F}_{1, n}+\widetilde{R}_{1, n}$ can be evaluated by Proposition 2.1 and reflection formulæ for $p, q \geq 2$ are given by

$$
\begin{aligned}
& \widetilde{F}_{p, q}+\widetilde{G}_{q, p}=N^{-w} \\
&\left\{\left(p, \frac{a}{N}\right) \zeta\left(q, \frac{3 a}{N}\right)+\zeta\left(p, \frac{N-a}{N}\right) \zeta\left(q, \frac{N-3 a}{N}\right)\right. \\
&\left.\quad-\zeta\left(p, \frac{2 a}{N}\right) \zeta\left(q, \frac{3 a}{N}\right)-\zeta\left(p, \frac{N-2 a}{N}\right) \zeta\left(q, \frac{N-3 a}{N}\right)\right\}
\end{aligned}
$$

and

$$
\begin{aligned}
\widetilde{R}_{p, q}-\widetilde{R}_{q, p} & =N^{-w}\left\{\zeta\left(p, \frac{a}{N}\right) \zeta\left(q, \frac{N-2 a}{N}\right)+\zeta\left(p, \frac{N-a}{N}\right) \zeta\left(q, \frac{2 a}{N}\right)\right. \\
& \left.-\zeta\left(p, \frac{2 a}{N}\right) \zeta\left(q, \frac{N-a}{N}\right)-\zeta\left(p, \frac{N-2 a}{N}\right) \zeta\left(q, \frac{a}{N}\right)\right\}
\end{aligned}
$$

Also we have the following relations among such triple families:

$$
\begin{aligned}
\widetilde{F}_{p, q} & =(-1)^{p+1} \sum_{l=2}^{q} B_{l} \widetilde{F}_{w-l, l}+(-1)^{p+1} A_{1} \widetilde{G}_{1, w-1}+\widetilde{f}_{p, q}, \\
\widetilde{G}_{p, q} & =(-1)^{p+1} \sum_{l=2}^{q} B_{l} \widetilde{R}_{w-l, l}+(-1)^{p} A_{1} \widetilde{R}_{1, w-1}+\widetilde{g}_{p, q}, \\
\widetilde{R}_{p, q} & =(-1)^{p+1} \sum_{l=2}^{q} B_{l} \widetilde{G}_{w-l, l}+(-1)^{p+1} A_{1} \widetilde{F}_{1, w-1}+\widetilde{r}_{p, q},
\end{aligned}
$$

where $\widetilde{f}_{p, q}, \widetilde{g}_{p, q}$ and $\widetilde{r}_{p, q}$ are known values in Hurwitz zeta functions.

Proposition 4.6. For each positive integer $n, \widetilde{F}_{1,2 n}, \widetilde{G}_{1,2 n}$ and $\widetilde{R}_{1,2 n}$ as defined in (4.20), (4.21) and (4.22), respectively, can be evaluated in terms of values at positive integers of Hurwitz zeta functions.

Proof. We already know the value of $\widetilde{F}_{1,2 n}+\widetilde{R}_{1,2 n}$. Now setting $p=2 n-1$ and $q=2$ in (4.25), (4.26) and (4.27), we get

$$
\left\{\begin{array}{l}
\widetilde{F}_{2 n-1,2}=\widetilde{F}_{2 n-1,2}-(2 n-1) \widetilde{G}_{1,2 n}+\widetilde{f}_{2 n-1,2}, \\
\widetilde{G}_{2 n-1,2}=\widetilde{R}_{2 n-1,2}+(2 n-1) \widetilde{R}_{1,2 n}+\widetilde{g}_{2 n-1,2}, \\
\widetilde{R}_{2 n-1,2}=\widetilde{G}_{2 n-1,2}-(2 n-1) \widetilde{F}_{1,2 n}+\widetilde{r}_{2 n-1,2} .
\end{array}\right.
$$

The value of $\widetilde{G}_{1,2 n}$ is obtained from the first equation, and the value of $\widetilde{F}_{1,2 n}-\widetilde{R}_{1,2 n}$ comes from the addition of the second and third equations. Thus our assertions follow. 
Once we know the values of $\widetilde{F}_{1,2 n}, \widetilde{G}_{1,2 n}$ and $\widetilde{R}_{1,2 n}$, we rewrite our relations in matrix form as before:

$$
\begin{aligned}
& \widetilde{F}=-B \widetilde{F}+\widetilde{\mathbf{f}}, \\
& \widetilde{G}=-B \widetilde{R}+\widetilde{\mathbf{g}}, \\
& \widetilde{R}=-B \widetilde{G}+\widetilde{\mathbf{r}}, \\
& \widetilde{F}+J \widetilde{G}=\mathbf{u}, \\
& \widetilde{R}-J \widetilde{R}=\mathbf{v},
\end{aligned}
$$

where the matrices $J$ and $B$ are given as before and $\widetilde{\mathbf{f}}, \widetilde{\mathbf{g}}, \widetilde{\mathbf{r}}, \mathbf{u}, \mathbf{v}$ are column vectors of size $(2 n-2) \times 1$ with known entries. From (1a), (2a) and (4a), we get

$$
(I+B) J B \widetilde{R}=(I+B)(J \widetilde{\mathbf{g}}-\mathbf{u})+\widetilde{\mathbf{f}} .
$$

Let $X=B \widetilde{R}$ be a new variable in place of $\widetilde{R}$. By a similar argument as before, the addition of (5a) and (6a) yields the system

$$
(J+B+B J-J B) X=(I+B)(J \widetilde{\mathbf{g}}-\mathbf{u})+\widetilde{\mathbf{f}}+\mathbf{v} .
$$

Then, again, the coefficient matrix $P=(J+B)-(J B-B J)$ is invertible since $P^{2}=2(J B+B J)$ has nonzero determinant by Corollary 3.4(i). This proves the following result.

THEOREM 4.7. For positive integers $j, k$ and $a$ with $k a<N$ and $k \geq$ $j+2, k \neq 2 j$, let

$$
\begin{aligned}
\widetilde{F}_{p, q}(j, k ; a, N):= & H_{p, q}\left(\frac{j a}{N}, \frac{k a}{N}\right)+H_{p, q}\left(\frac{N-j a}{N}, \frac{N-k a}{N}\right) \\
& -H_{p, q}\left(\frac{(k-j) a}{N}, \frac{k a}{N}\right)-H_{p, q}\left(\frac{N-(k-j) a}{N}, \frac{N-k a}{N}\right), \\
\widetilde{G}_{p, q}(j, k ; a, N):= & H_{p, q}\left(\frac{k a}{N}, \frac{j a}{N}\right)+H_{p, q}\left(\frac{N-k a}{N}, \frac{N-j a}{N}\right) \\
& -H_{p, q}\left(\frac{k a}{N}, \frac{(k-j) a}{N}\right)-H_{p, q}\left(\frac{N-k a}{N}, \frac{N-(k-j) a}{N}\right), \\
\widetilde{R}_{p, q}(j, k ; a, N):= & H_{p, q}\left(\frac{j a}{N}, \frac{N-(k-j) a}{N}\right)+H_{p, q}\left(\frac{N-j a}{N}, \frac{(k-j) a}{N}\right) \\
& -H_{p, q}\left(\frac{(k-j) a}{N}, \frac{N-j a}{N}\right)-H_{p, q}\left(\frac{N-(k-j) a}{N}, \frac{j a}{N}\right) .
\end{aligned}
$$

Then $\widetilde{F}_{p, q}(j, k ; a, N), \widetilde{G}_{p, q}(j, k ; a, N)$ and $\widetilde{R}_{p, q}(j, k ; a, N)$ can be evaluated in terms of values at positive integers of Hurwitz zeta functions when the weight $p+q$ is odd. 
Combining Theorem 4.3, its corollaries as above and Theorem 4.7, we obtain a much powerful theorem.

THEOREM 4.8. For a pair of positive integers $a$ and $b$ with $a, b<N$, the sum defined by

$$
H_{p, q}\left(\frac{a}{N}, \frac{b}{N}\right)+H_{p, q}\left(\frac{N-a}{N}, \frac{N-b}{N}\right)
$$

can be evaluated in terms of values at positive integers of Hurwitz zeta functions when the weight $p+q$ is odd.

Now we are ready to prove the first part of the Main Theorem.

Proof of the Main Theorem for even characters. When the character $\chi$ is even, we rewrite $S_{p, q}^{\chi}$ as

$$
2 S_{p, q}^{\chi}=\sum_{b=1}^{N-1} \chi(b) \sum_{a=1}^{N}\left\{H_{p, q}\left(\frac{a}{N}, \frac{b}{N}\right)+H_{p, q}\left(\frac{a}{N}, \frac{N-b}{N}\right)\right\} .
$$

The inner summation can be rearranged as

$$
\begin{aligned}
\sum_{a=1}^{N-1}\left\{H_{p, q}\left(\frac{a}{N}, \frac{b}{N}\right)+H_{p, q}(\right. & \left.\left.\frac{N-a}{N}, \frac{N-b}{N}\right)\right\} \\
& +\left\{H_{p, q}\left(\frac{N}{N}, \frac{b}{N}\right)+H_{p, q}\left(\frac{N}{N}, \frac{N-b}{N}\right)\right\} .
\end{aligned}
$$

Consequently, $S_{p, q}^{\chi}$ can be evaluated when the weight $w=p+q$ is odd, by Theorem 4.8 and Corollary 4.4.

5. Euler sums with odd characters. When the character $\chi$ is odd, we rewrite $S_{p, q}^{\chi}$ as

$$
2 S_{p, q}^{\chi}=\sum_{b=1}^{N-1} \chi(b) \sum_{a=1}^{N}\left\{H_{p, q}\left(\frac{a}{N}, \frac{b}{N}\right)-H_{p, q}\left(\frac{a}{N}, \frac{N-b}{N}\right)\right\} .
$$

We shall henceforth fix the weight $w=p+q=2 n+2$ with a positive integer $n$ and matrices $I, B, J$ as before except the size is $(2 n-1) \times(2 n-1)$.

Consider the new triple families defined by

$$
\begin{aligned}
K_{p, q}^{-}(a, N) & :=H_{p, q}\left(\frac{a}{N}, \frac{2 a}{N}\right)-H_{p, q}\left(\frac{N-a}{N}, \frac{N-2 a}{N}\right), \\
L_{p, q}^{-}(a, N) & :=H_{p, q}\left(\frac{2 a}{N}, \frac{a}{N}\right)-H_{p, q}\left(\frac{N-2 a}{N}, \frac{N-a}{N}\right), \\
M_{p, q}^{-}(a, N) & :=H_{p, q}\left(\frac{a}{N}, \frac{N-a}{N}\right)-H_{p, q}\left(\frac{N-a}{N}, \frac{a}{N}\right) .
\end{aligned}
$$


We use the notations $K_{p, q}^{-}, L_{p, q}^{-}$and $M_{p, q}^{-}$instead of $K_{p, q}^{-}(a, N), L_{p, q}^{-}(a, N)$ and $M_{p, q}^{-}(a, N)$, respectively. With similar arguments as before, we obtain the following relations for $p, q \geq 2$ :

$$
\begin{aligned}
K_{p, q}^{-} & =(-1)^{p} \sum_{l=2}^{q} B_{l} K_{w-l, l}^{-}+(-1)^{p} A_{1} L_{1, w-1}^{-}+k_{p, q}^{-}, \\
L_{p, q}^{-} & =(-1)^{p+1} \sum_{l=2}^{q} B_{l} M_{w-l, l}^{-}+(-1)^{p} A_{1} M_{1, w-1}^{-}+\ell_{p, q}^{-}, \\
M_{p, q}^{-} & =(-1)^{p+1} \sum_{l=2}^{q} B_{l} L_{w-l, l}^{-}+(-1)^{p+1} A_{1} K_{1, w-1}^{-}+m_{p, q}^{-}
\end{aligned}
$$

The reflection formulæ for such families are given as follows:

$$
\begin{aligned}
& K_{p, q}^{-}+L_{q, p}^{-} \\
& \quad=N^{-w}\left\{\zeta\left(p, \frac{a}{N}\right) \zeta\left(q, \frac{2 a}{N}\right)-\zeta\left(p, \frac{N-a}{N}\right) \zeta\left(q, \frac{N-2 a}{N}\right)\right\}, \\
& M_{p, q}^{-}-M_{q, p}^{-} \\
& \quad=N^{-w}\left\{\zeta\left(p, \frac{a}{N}\right) \zeta\left(q, \frac{N-a}{N}\right)-\zeta\left(p, \frac{N-a}{N}\right) \zeta\left(q, \frac{a}{N}\right)\right\} .
\end{aligned}
$$

Also note that

$$
\begin{aligned}
K_{1, w-1}^{-}- & M_{1, w-1}^{-}=\left\{H_{1, w-1}\left(\frac{a}{N}, \frac{2 a}{N}\right)+H_{1, w-1}\left(\frac{N-a}{N}, \frac{a}{N}\right)\right\} \\
& -\left\{H_{1, w-1}\left(\frac{N-a}{N}, \frac{N-2 a}{N}\right)+H_{1, w-1}\left(\frac{a}{N}, \frac{N-a}{N}\right)\right\},
\end{aligned}
$$

which can be evaluated by Proposition 2.2.

Now we begin with the evaluation for $p=1$ and $q=2 n+1$.

Proposition 5.1. For each positive integer $n, K_{1,2 n+1}^{-}, L_{1,2 n+1}^{-}$and $M_{1,2 n+1}^{-}$can be evaluated in terms of values at positive integers of Hurwitz zeta functions.

Proof. Setting $p=2 n$ and $q=2$ in (5.5), we get

$$
K_{2 n, 2}^{-}=K_{2 n, 2}^{-}-2 n L_{1,2 n+1}^{-}+k_{2 n, 2}^{-} \text {. }
$$

Thus $L_{1,2 n+1}^{-}$can be evaluated. On the other hand, setting $p=2 n$ and $q=2$ in (5.6) and (5.7), we get

$$
\begin{gathered}
L_{2 n, 2}^{-}=-M_{2 n, 2}^{-}-2 n M_{1,2 n+1}^{-}+\ell_{2 n, 2}^{-}, \\
M_{2 n, 2}^{-}=-L_{2 n, 2}^{-}+2 n K_{1,2 n+1}^{-}+m_{2 n, 2}^{-} .
\end{gathered}
$$

It follows that

$$
2 n\left\{K_{1,2 n+1}^{-}+M_{1,2 n+1}^{-}\right\}=-m_{2 n, 2}^{-}+\ell_{2 n, 2}^{-} .
$$


The evaluations of $K_{1,2 n+1}^{-}$and $M_{1,2 n+1}^{-}$can be obtained by (5.10) and (5.14).

Once we get the values of $K_{1,2 n+1}^{-}, L_{1,2 n+1}^{-}$and $M_{1,2 n+1}^{-}$, we rewrite our system of equations in unknowns $K_{2,2 n}^{-}, K_{3,2 n-1}^{-}, \ldots, K_{2 n, 2}^{-} ; L_{2,2 n}^{-}, L_{3,2 n-1}^{-}$, $\ldots, L_{2 n, 2}^{-} ; M_{2,2 n}^{-}, M_{3,2 n-1}^{-}, \ldots, M_{2 n, 2}^{-}$in matrix form as follows:

$$
\begin{aligned}
& K^{-}=B K^{-}+\mathbf{k}^{-}, \\
& L^{-}=-B M^{-}+\mathbf{l}^{-}, \\
& M^{-}=-B L^{-}+\mathbf{m}^{-}, \\
& K^{-}+J L^{-}=\mathbf{c}^{-}, \\
& M^{-}-J M^{-}=\mathbf{d}^{-},
\end{aligned}
$$

where $\mathbf{k}^{-}, \mathbf{l}^{-}, \mathbf{m}^{-}, \mathbf{c}^{-}, \mathbf{d}^{-}$are $(2 n-1) \times 1$ column vectors with known values. Again we have

$$
(I-B) J B M^{-}=\mathbf{k}^{-}-(I-B)\left(\mathbf{c}^{-}-J 1^{-}\right) .
$$

The rank of $(I-B) J B$ is $n-1$ and the condition (5b) provides another $n-1$ equations. All these relations are still not enough to determine the value of $M^{-}$. However, we have an extra relation from $L_{1,2 n+1}^{-}$:

$$
L_{1,2 n+1}^{-}=M_{1,2 n+1}^{-}+\sum_{l=1}^{2 n} M_{l, 2 n+2-l}+\ell_{1,2 n+1}^{-} .
$$

This gives the final condition needed so that we can solve for $M$ by (5b), (6b) and (7b).

Here we show the solvability of the system. Let $X=B M^{-}$be a new variable in place of $M^{-}$, and $W$ be the $(2 n-1) \times(2 n-1)$ matrix with all entries 1 . Then (5b), (6b) and (7b) are equivalent to the system

$$
\left\{\begin{array}{l}
(B-J B) X=\mathbf{d}^{-}, \\
(J-B J) X=\mathbf{k}^{-}-(I-B)\left(\mathbf{c}^{-}-J \mathbf{l}^{-}\right), \\
W X=\mathbf{w}^{-},
\end{array}\right.
$$

where $\mathbf{w}^{-}$is a $(2 n-1) \times 1$ column vector with every entry equal to $L_{1,2 n+1}^{-}$ $2 M_{1,2 n+1}^{-}-\ell_{1,2 n+1}^{-}$. Consider the sum of the first two equations. The coefficient matrix of the resulting equation is $J+B-J B-B J$, which we denote by $P$. Note that $B^{2}=J^{2}=I$ and hence

$$
P=-(J+B+I)(J+B-2 I) .
$$

Now we let $\widetilde{P}=P-(J+B+I) W=-(J+B+I)(J+B-2 I+W)$, the coefficient matrix of a combination of the system. To prove $\widetilde{P}$ is invertible, it suffices to prove that both $J+B+I$ and $J+B-2 I+W$ are invertible. First we prove that $J+B+I$ is invertible. By Corollary 3.4(ii), the eigenvalues 
of $J+B$ lie in the set $\{0, \pm 2, \pm \sqrt{3}\}$. It follows that $J+B+I$ is invertible since its eigenvalues are among $\pm 1,3,1 \pm \sqrt{3}$, none of which is zero. Next we prove that $J+B-2 I+W$ is also invertible. Suppose that $\mathbf{h}$ is a $(2 n-1) \times 1$ column vector such that

$$
(J+B-2 I+W) \mathbf{h}=0 .
$$

Applying $W$ to the above equality and noting that $W J=W, W B=W$, we get

$$
W^{2} \mathbf{h}=0 \quad \text { or } \quad(2 n-1) W \mathbf{h}=0 .
$$

Hence $W \mathbf{h}=0$ and $(J+B) \mathbf{h}=2 \mathbf{h}$. A direct calculation yields

$$
J B \mathbf{h}=3 \mathbf{h}-2 B \mathbf{h}, \quad B J \mathbf{h}=-\mathbf{h}+2 B \mathbf{h}
$$

and

$$
(J B)^{2} \mathbf{h}=5 \mathbf{h}-4 B \mathbf{h}, \quad(B J)^{2} \mathbf{h}=-3 \mathbf{h}+4 B \mathbf{h} .
$$

The identity $(J B)^{2}-(B J)^{2}=J B-B J$ then implies that $B \mathbf{h}=\mathbf{h}$. Now from

$$
W \mathbf{h}=0, \quad B \mathbf{h}=\mathbf{h}, \quad J \mathbf{h}=\mathbf{h},
$$

we get $\mathbf{h}=0$ since the ranks of $W, B-I$ and $J-I$ are $1, n-1$ and $n-1$, respectively. Thus $J+B-2 I+W$ is invertible. We have thus proved

ThEOREM 5.2. For a positive integer a with $2 a<N$, let $K_{p, q}^{-}(a, N)$, $L_{p, q}^{-}(a, N)$ and $M_{p, q}^{-}(a, N)$ be defined as in (5.2), (5.3) and (5.4). Then they can be evaluated in terms of values at positive integers of Hurwitz zeta functions when the weight $p+q$ is even.

By exactly the same procedure, we obtain the following theorem.

THEOREM 5.3. Suppose that $j, k, a$ are positive integers such that $k a<N$ and $k \geq j+2, k \neq 2 j$ and

$$
\begin{aligned}
& F_{p, q}^{-}(j, k ; a, N):=H_{p, q}\left(\frac{j a}{N}, \frac{k a}{N}\right)+H_{p, q}\left(\frac{(k-j) a}{N}, \frac{k a}{N}\right) \\
&-H_{p, q}\left(\frac{N-j a}{N}, \frac{N-k a}{N}\right)-H_{p, q}\left(\frac{N-(k-j) a}{N}, \frac{N-k a}{N}\right), \\
& G_{p, q}^{-}(j, k ; a, N):=H_{p, q}\left(\frac{k a}{N}, \frac{j a}{N}\right)+H_{p, q}\left(\frac{k a}{N}, \frac{(k-j) a}{N}\right) \\
&-H_{p, q}\left(\frac{N-k a}{N}, \frac{N-j a}{N}\right)-H_{p, q}\left(\frac{N-k a}{N}, \frac{N-(k-j) a}{N}\right),
\end{aligned}
$$

$$
\begin{array}{r}
R_{p, q}^{-}(j, k ; a, N):=H_{p, q}\left(\frac{j a}{N}, \frac{N-(k-j) a}{N}\right)+H_{p, q}\left(\frac{(k-j) a}{N}, \frac{N-j a}{N}\right) \\
-H_{p, q}\left(\frac{N-(k-j) a}{N}, \frac{j a}{N}\right)-H_{p, q}\left(\frac{N-j a}{N}, \frac{(k-j) a}{N}\right) .
\end{array}
$$


Then $F_{p, q}^{-}(j, k ; a, N), G_{p, q}^{-}(j, k ; a, N)$ and $R_{p, q}^{-}(j, k ; a, N)$ can be evaluated in terms of values at positive integers of Hurwitz zeta functions when the weight $w=p+q$ is even.

Triple families with four members as above can be separated further into subfamilies with just two members. The argument is similar to that for odd weight, so we omit it here.

THEOREM 5.4. Suppose that $j$ and $k$ are positive integers such that $k a<N, k \geq j+2, k \neq 2 j$ and

$$
\begin{aligned}
\widetilde{F}_{p, q}^{-}(j, k ; a, N):= & \left.H_{p, q}\left(\frac{j a}{N}, \frac{k a}{N}\right)-H_{p, q}\left(\frac{N-j a}{N}, \frac{N-k a}{N}\right)\right\} \\
& -\left\{H_{p, q}\left(\frac{(k-j) a}{N}, \frac{k a}{N}\right)-H_{p, q}\left(\frac{N-(k-j) a}{N}, \frac{N-k a}{N}\right)\right\}, \\
\widetilde{G}_{p, q}^{-}(j, k ; a, N):= & \left\{H_{p, q}\left(\frac{k a}{N}, \frac{j a}{N}\right)-H_{p, q}\left(\frac{N-k a}{N}, \frac{N-j a}{N}\right)\right\} \\
& -\left\{H_{p, q}\left(\frac{k a}{N}, \frac{(k-j) a}{N}\right)-H_{p, q}\left(\frac{N-k a}{N}, \frac{N-(k-j) a}{N}\right)\right\}, \\
\widetilde{R}_{p, q}^{-}(j, k ; a, N):= & \left\{H_{p, q}\left(\frac{j a}{N}, \frac{N-(k-j) a}{N}\right)-H_{p, q}\left(\frac{N-j a}{N}, \frac{(k-j) a}{N}\right)\right\} \\
& -\left\{H_{p, q}\left(\frac{(k-j) a}{N}, \frac{N-j a}{N}\right)-H_{p, q}\left(\frac{N-(k-j) a}{N}, \frac{j a}{N}\right)\right\} .
\end{aligned}
$$

Then $\widetilde{F}_{p, q}^{-}(j, k ; a, N), \widetilde{G}_{p, q}^{-}(j, k ; a, N)$ and $\widetilde{R}_{p, q}^{-}(j, k ; a, N)$ can be evaluated in terms of values at positive integers of Hurwitz zeta functions when the weight $w=p+q$ is even.

We can combine Theorems 5.2, 5.3 and 5.4 into the following more general theorem.

THEOREM 5.5. For a pair of positive integers $a$ and $b$ with $a \neq b$ and $a, b<N$, the sum defined by

$$
H_{p, q}\left(\frac{a}{N}, \frac{b}{N}\right)-H_{p, q}\left(\frac{N-a}{N}, \frac{N-b}{N}\right)
$$

can be evaluated in terms of values at positive integers of Hurwitz zeta functions when the weight $p+q$ is even.

There is a slight difference for the triple families defined by

$$
E_{p, q}^{-}(a, N):=H_{p, q}\left(\frac{a}{N}, \frac{N}{N}\right)-H_{p, q}\left(\frac{N-a}{N}, \frac{N}{N}\right)
$$




$$
\begin{aligned}
T_{p, q}^{-}(a, N) & :=H_{p, q}\left(\frac{N}{N}, \frac{a}{N}\right)-H_{p, q}\left(\frac{N}{N}, \frac{N-a}{N}\right) \\
D_{p, q}^{-}(a, N) & :=H_{p, q}\left(\frac{a}{N}, \frac{a}{N}\right)-H_{p, q}\left(\frac{N-a}{N}, \frac{N-a}{N}\right) .
\end{aligned}
$$

Again, we simplify the notations as $E_{p, q}^{-}, T_{p, q}^{-}$and $D_{p, q}^{-}$. With the same argument as before, we get the following relations for $p, q \geq 2$ :

$$
\begin{aligned}
& E_{p, q}^{-}=(-1)^{p+1} \sum_{l=2}^{q} B_{l} E_{w-l, l}^{-}+(-1)^{p+1} A_{1} T_{1, w-1}^{-}+e_{p, q}, \\
& T_{p, q}^{-}=(-1)^{p} \sum_{l=2}^{q} B_{l} D_{w-l, l}^{-}+(-1)^{p} A_{1} D_{1, w-1}^{-}+t_{p, q}, \\
& D_{p, q}^{-}=(-1)^{p} \sum_{l=2}^{q} B_{l} T_{w-l, l}^{-}+(-1)^{p} A_{1} E_{1, w-1}^{-}+d_{p, q} .
\end{aligned}
$$

The reflection formulæ for such families are

$$
\begin{aligned}
& E_{p, q}^{-}+T_{q, p}^{-}=N^{-w}\left\{\zeta\left(p, \frac{a}{N}\right) \zeta(q)-\zeta\left(p, \frac{N-a}{N}\right) \zeta(q)\right\} \\
& D_{p, q}^{-}+D_{q, p}^{-} \\
& \quad=N^{-w}\left\{\zeta\left(p, \frac{a}{N}\right) \zeta\left(q, \frac{a}{N}\right)-\zeta\left(p, \frac{N-a}{N}\right) \zeta\left(q, \frac{N-a}{N}\right)\right\} .
\end{aligned}
$$

Furthermore, we note that

$$
\begin{aligned}
E_{1, n}^{-}-D_{1, n}^{-}= & \left\{H_{1, n}\left(\frac{N-a}{N}, \frac{N-a}{N}\right)+H_{1, n}\left(\frac{a}{N}, \frac{N}{N}\right)\right\} \\
& -\left\{H_{1, n}\left(\frac{a}{N}, \frac{a}{N}\right)+H_{1, n}\left(\frac{N-a}{N}, \frac{N}{N}\right)\right\},
\end{aligned}
$$

which can be evaluated by Proposition 2.3. Also $T_{1, n}^{-}$can be evaluated by Proposition 2.1.

Proposition 5.6. For each positive integer $n, E_{1,2 n+1}^{-}, T_{1,2 n+1}^{-}$and $D_{1,2 n+1}^{-}$can be evaluated in terms of values at positive integers of Hurwitz zeta functions.

Proof. It suffices to evaluate $E_{1,2 n+1}^{-}$and $D_{1,2 n+1}^{-}$. However, their difference given by (5.26) is known. Setting $p=2 n$ and $q=2$ in (5.22) and (5.23), we get

$$
\begin{aligned}
T_{2 n, 2}^{-} & =D_{2 n, 2}^{-}-2 n D_{1,2 n+1}^{-}+t_{2 n, 2}, \\
D_{2 n, 2}^{-} & =T_{2 n, 2}^{-}-2 n E_{1,2 n+1}^{-}+d_{2 n, 2} .
\end{aligned}
$$


It follows that

$$
2 n\left\{E_{1,2 n+1}^{-}+D_{1,2 n+1}^{-}\right\}=t_{2 n, 2}+d_{2 n, 2} \text {. }
$$

Thus the evaluations of $E_{1,2 n+1}^{-}$and $D_{1,2 n+1}^{-}$follow from (5.26) and (5.27).

Now we rewrite (5.21) to (5.25) in matrix form with unknowns

$$
\begin{aligned}
& E^{-}={ }^{t}\left(E_{2,2 n}^{-}, E_{3,2 n-1}^{-}, \ldots, E_{2 n, 2}^{-}\right), \\
& T^{-}={ }^{t}\left(T_{2,2 n}^{-}, T_{3,2 n-1}^{-}, \ldots, T_{2 n, 2}^{-}\right), \\
& D^{-}={ }^{t}\left(D_{2,2 n}^{-}, D_{3,2 n-1}^{-}, \ldots, D_{2 n, 2}^{-}\right) .
\end{aligned}
$$

The system of equations are given as follows:

$$
\begin{aligned}
& E^{-}=-B E^{-}+\mathbf{e}^{-}, \\
& T^{-}=B D^{-}+\mathbf{t}^{-}, \\
& D^{-}=B T^{-}+\mathbf{d}^{-}, \\
& E^{-}+J T^{-}=\mathbf{u}^{-} \\
& D^{-}+J D^{-}=\mathbf{v}^{-}
\end{aligned}
$$

where $\mathbf{e}^{-}, \mathbf{t}^{-}, \mathbf{d}^{-}, \mathbf{u}^{-}, \mathbf{v}^{-}$are $(2 n-1) \times 1$ column vectors with known entries.

With a similar argument as before, we get the equation

$$
\{(I+B) J B+I+J\} D^{-}=(I+B)\left(\mathbf{u}-J \mathbf{t}^{-}\right)-\mathbf{e}^{-}
$$

from (1c), (2c), (4c) and (5c). Let $P=(I+B) J B+I+J$. Then

$$
\begin{aligned}
P B & =J+B+B J+J B \\
& =(J+B)^{2}+(J+B)-2 I \\
& =(J+B-I)(J+B+2 I) .
\end{aligned}
$$

By a similar argument to the proof of Theorem 5.2, we see that both $J+B-I$ and $J+B+2 I$ are invertible, and thus the following theorem is proved.

THEOREM 5.7. Suppose that $a$ is a positive integer with $a<N$ and $E_{p, q}^{-}(a, N), T_{p, q}^{-}(a, N)$, and $D_{p, q}^{-}(a, N)$ are defined as in (5.18), (5.19), and (5.20), respectively. Then $E_{p, q}^{-}(a, N), T_{p, q}^{-}(a, N)$, and $D_{p, q}^{-}(a, N)$ can be evaluated in terms of values at positive integers of Hurwitz zeta functions when the weight $w=p+q$ is even.

Now we prove the remaining half of the Main Theorem. 
Completion of the proof of the Main Theorem. Suppose $\chi$ is odd and $p+q$ is even. As mentioned earlier, we rewrite

$$
2 S_{p, q}^{\chi}=\sum_{b=1}^{N-1} \chi(b) \sum_{a=1}^{N}\left\{H_{p, q}\left(\frac{a}{N}, \frac{b}{N}\right)-H_{p, q}\left(\frac{a}{N}, \frac{N-b}{N}\right)\right\} .
$$

The inner summation can be rearranged as

$$
\begin{aligned}
\sum_{a=1}^{N-1}\left\{H_{p, q}\left(\frac{a}{N}, \frac{b}{N}\right)-H_{p, q}(\right. & \left.\left.\frac{N-a}{N}, \frac{N-b}{N}\right)\right\} \\
& +\left\{H_{p, q}\left(\frac{N}{N}, \frac{b}{N}\right)-H_{p, q}\left(\frac{N}{N}, \frac{N-b}{N}\right)\right\} .
\end{aligned}
$$

Thus our assertions follow from Theorems 5.5 and 5.7.

As an immediate consequence of our Main Theorem, we have

Corollary 5.8. For a pair of positive integers $p$ and $q$ with $q \geq 2$ and a Dirichlet character $\chi$ of conductor $N$, let

$$
\bar{S}_{p, q}^{\chi}:=\sum_{k=1}^{\infty} \frac{1}{k^{q}} \sum_{j=1}^{k} \frac{\chi(j)}{j^{p}} .
$$

Then $\bar{S}_{p, q}^{\chi}$ can be evaluated in terms of Hurwitz zeta functions when either $\chi$ is even and $p+q$ is odd, or $\chi$ is odd and $p+q$ is even.

Concluding remarks. 1. Although the Main Theorem was only stated for the sum $S_{p, q}^{\chi}$, our methods are in fact applicable to the evaluation of the most general double Euler sums with Dirichlet characters of the form

$$
S_{p, q}^{\chi_{m}, \chi_{n}}:=\sum_{k=1}^{\infty} \frac{\chi_{n}(k)}{k^{q}} \sum_{j=1}^{k} \frac{\chi_{m}(j)}{j^{p}}
$$

where $\chi_{m}$ and $\chi_{n}$ are Dirichlet characters modulo $m$ and $n$, respectively. Putting $N=\operatorname{lcm}\{m, n\}$, we can rewrite the sum as

$$
\begin{aligned}
\frac{1}{2} \sum_{a=1}^{N-1} \sum_{b=1}^{N-1} \chi_{m}(a) \chi_{n}(b)\left\{H_{p, q}\right. & \left(\frac{a}{N}, \frac{b}{N}\right) \\
& \left.+\chi_{m}(-1) \chi_{n}(-1) H_{p, q}\left(\frac{N-a}{N}, \frac{N-b}{N}\right)\right\} .
\end{aligned}
$$

Analyzing the inner sum $H_{p, q}(a / N, b / N) \pm H_{p, q}((N-a) / N,(N-b) / N)$ as we did in this paper, one can deduce that if $\chi_{m}(-1) \chi_{n}(-1)=1$, then the general sum may be evaluated in terms of values at positive integers of Hurwitz zeta functions when the weight $p+q$ is odd, while when $\chi_{m}(-1) \chi_{n}(-1)$ $=-1$, the evaluation may be obtained when $p+q$ is even. 
2. The same analysis that allows us to prove the invertibility of various matrices can be used to obtain the minimum polynomial of the coefficient matrix $P$ for the linear systems treated in this paper. The inverse of $P$ is therefore explicitly given and one can then solve the associated system to effect an evaluation of various Euler sums. However, we should remark that the evaluation obtained this way is not very tractable, as was also noted by Borwein et al. [2, p. 293].

Acknowledgments. The authors thank the referee for helpful suggestions, which improved the paper substantially.

\section{References}

[1] B. C. Berndt, Ramanujan's Notebooks, Part I, Springer, New York, 1985.

[2] D. Borwein, J. M. Borwein, and R. Girgensohn, Explicit evaluation of Euler sums, Proc. Edinburgh Math. Soc. (2) 38 (1995), 277-294.

[3] J. M. Borwein, D. M. Bradley, and D. J. Broadhurst, Evaluation of k-fold Euler/ Zagier sums: a compendium of results for arbitrary $k$, Electron. J. Combin. 4 (1997), no. 5 .

[4] J. M. Borwein and R. Girgensohn, Evaluation of triple Euler sums, Electron. J. Combin. 3 (1996), no. 1, \#R 23, 27 pp., with an appendix by D. J. Broadhurst, Euler sums in quantum field theory.

[5] J. M. Borwein, I. J. Zuckr, and J. Boersma, The evaluation of character Euler double sums, Ramanujan J. (2007), to appear.

[6] R. E. Crandall and J. P. Buhler, On the evaluation of Euler sums, Experiment. Math. 3 (1994), 275-285.

[7] K.-W. Chen and M. Eie, Explicit evaluations of extended Euler sums, J. Number Theory 117 (2006), 31-52.

[8] M. Eie, W.-C. Liaw, and Y. L. Ong, Euler sums on arithmetic progressions, New Zealand J. Math., to appear.

[9] M. Eie, W.-C. Liaw, and F.-Y. Yang, On evaluations of generalized Euler sums of even weight, Internat. J. Number Theory 1 (2005), 225-242.

[10] L. Euler, Meditationes circa singulare serierum genus, Novi Comm. Acad. Sci. Petropol. 20 (1775), 140-186. Reprinted in Opera Omnia, ser. 1, vol. XV, Teubner, Berlin, 1917, 217-267.

[11] P. Flajolet and B. Salvy, Euler sums and contour integral representations, Experiment. Math. 7 (1998), 15-35.

[12] C. Markett, Triple sums and the Riemann zeta function, J. Number Theory 48 (1994), 113-132.

[13] N. Nielsen, Handbuch der Theorie der Gammafunktion and Theorie des Integrallogarithmus und verwandter Transzendenten, 1906. Reprinted together as Die Gammafunktion, Chelsea, New York, 1965.

[14] D. Terhune, Evaluation of double L-values, J. Number Theory 105 (2004), 275-301.

[15] - Evaluations of a class of double L-values, Proc. Amer. Math. Soc. 134 (2006), 1881-1889.

[16] L. Tornheim, Harmonic double series, Amer. J. Math. 72 (1950), 303-314. 
[17] L. C. Washington, Introduction to Cyclotomic Fields, 2nd ed., Springer, New York, 1997.

[18] E. T. Whittaker and G. N. Watson, A Course of Modern Analysis, 4th ed., Cambridge Univ. Press, Cambridge, 1927. Reprinted 1996.

[19] D. Zagier, Values of zeta functions and their generalizations, in: A. Joseph et al. (eds.), First European Congress of Mathematics, Paris, 1992, Vol. II, Progr. Math. 120, Birkhäuser, Basel, 1994, 487-512.

Department of Mathematics

National Chung Cheng University

Minhsiung, Chiayi 62145, Taiwan

E-mail: minking@math.ccu.edu.tw

wcliaw@math.ccu.edu.tw

Received on 27.6.2006

and in revised form on 23.4.2007 